See discussions, stats, and author profiles for this publication at: https://www.researchgate.net/publication/282246274

\title{
Off-line Signature Stability by Optical Flow: Feasibility Study of Predicting the Verifier Performance
}

Conference Paper · September 2015

DOI: $10.1109 /$ CCST.2015.7389707

\section{CITATIONS}

7

6 authors, including:

Moises Diaz

Universidad del Atlantico Medio

62 PUBLICATIONS 474 CITATIONS

SEE PROFILE

Giuseppe Pirlo

Università degli Studi di Bari Aldo Moro

209 PUBLICATIONS 2,166 CITATIONS

SEE PROFILE
READS

99

Miguel A. Ferrer

Universidad de Las Palmas de Gran Canaria

273 PUBLICATIONS 2,281 CITATIONS

SEE PROFILE

(f. Patricia Henríquez Rodríguez

Universidad de Las Palmas de Gran Canaria

25 PUBLICATIONS 289 CITATIONS

SEE PROFILE

Some of the authors of this publication are also working on these related projects:

Project Application of the Lognormal Model to the Vocal Tract Movement View project

Project sclera biometrics View project 


\title{
Off-line Signature Stability by Optical Flow: Feasibility Study of Predicting the Verifier Performance
}

\author{
Moises Diaz*, Miguel A. Ferrer*, Giuseppe Pirlo $^{\dagger}$ Giuliano Giannico $^{\dagger}$, Patricia Henriquez* ${ }^{*}$ and Donato Impedovo ${ }^{\ddagger}$ \\ IDETIC - Universidad de Las Palmas de Gran Canaria, Campus de Tafira s/n, E35017, Las Palmas de Gran Canaria, Spain. \\ Email: \{mdiaz, mferrer, phenriquez $\}$ idetic.eu \\ †'Dipartimento di Informatica, Università degli Studi di Bari "Aldo Moro", Via Orabona, 4, 70125 Bari, Italy \\ Email: giuseppe.pirlo@uniba.it, giulianogiannico@gmail.com \\ †DyrectaLab, Italy. Email: impedovo@gmail.com
}

\begin{abstract}
Prediction of performance in Off-line Automatic Signature Verification (ASV) per signer is one of the important topics regarding to automatic verification. It could be hypothesized that the performance of a signer is related to its global stability. This way, the more stable the signer signatures, the smaller the area of its feature space is, being more difficult to get inside for an impostor. In this paper we assess the feasibility to predict the performance of a signer through his/her global stability. As in a real scenario, only the enrolled signatures are used to calculate the stability of the signer. Similarly, only these signatures are used to train two completely different off-line ASVs. Then, the performance and the stability per signer are compared. Our results suggest that there is a certain relationship between the global stability of the enrolled signatures and the performance in terms of Equal Error Rate.
\end{abstract}

\section{INTRODUCTION}

The ASV " $A$ " works better for user "001" than user "002", however the ASV " $B$ " is more adequate for the signatures of the user "002". This is a good knowledge that the systems should be able to predict by using only the enrolled signatures without using the test set. Apart from the emerging aspects [1], the performance prediction of the individuals is an active problem in off-line signature verification. It is supposed to classify better the signatures according to their behavior, typically designed as Sheep, Lambs, Goats and Wolves categories [2].

Looking at signature-based images, a human inspection could detect a certain intra-personal variability in the lexical and morphology aspects [3] among the genuine signatures of a signer. This variability is closely related to the stability of the signature, assuming that the more intra-personal variability, the less stable a signer is. For instance, Fig. 1 highlights the overlapped signatures of two users with different stability levels: While the left signer has a more stable profile, the right one presents a more confusing and less stable profile.

Generally, it is studied that the "quality" of a biometric trait is related to the performance of the verifiers [4]. Particularly, in off-line signatures has been previously studied the relationship between signature quality and performances [5]. Authors used two scalar parameters denoted as slant and variability measurement. Also, another two terms so called "legibility" and "type" of a signature have been studied with off-line specimens, which

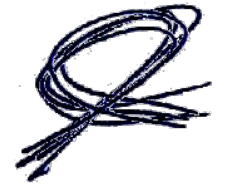

(a) Signer \#1

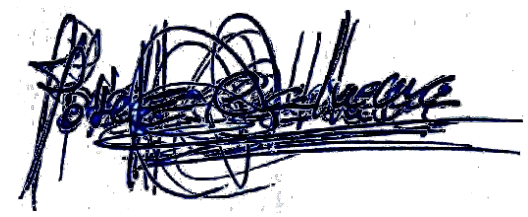

(b) Signer \#2
Fig. 1: Two signers with different global stabilities.

seems to have some connection with their performance in [6]. Although such advances have been carried out, there is still room to extract more fidelity information from the signatures used in the training in order to predict its performance.

In this work we propose the "stability" as a new concept which keeps a certain relationship with the performance. This way, the approach proposed by Horn and Shunck [7] based on optical flow has been considered to obtain a stability map with the enrolled signatures of a signer. Then, both the energy and the entropy of these maps are calculated to quantify the stability of a signer. Two state-of-the-art automatic off-line signature verifiers are worked out in order to evaluate the performance of each signer. Our results suggest that the stability interpreters are feasible to estimate a priori the performance by only using the enrolled signatures in a system.

The rest of this paper is organized as follows: Sect. II introduces the optical flow algorithm used in off-line signatures. While Sect. III describes the proposal methodology to compute the stability scalar values per user, Sect. IV explains the systems used to calculate the performances. The association between stability and performance in off-line signatures is given in V. The paper ends with the conclusions in Sect. VI.

\section{OPTICAL FlOW FOR STATIC Signatures}

Optical flow is a well-known technique to detect the movement of an object considering the relative motion between the observer and the object. This technique is widely used in computer vision systems such as object tracking, motion detection, image segmentation and stabilization. Many authors have approached the optical flow problem in the literature: region- 
Scanned Signatures
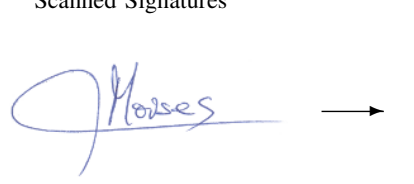

Local Stability Maps $S_{i}$
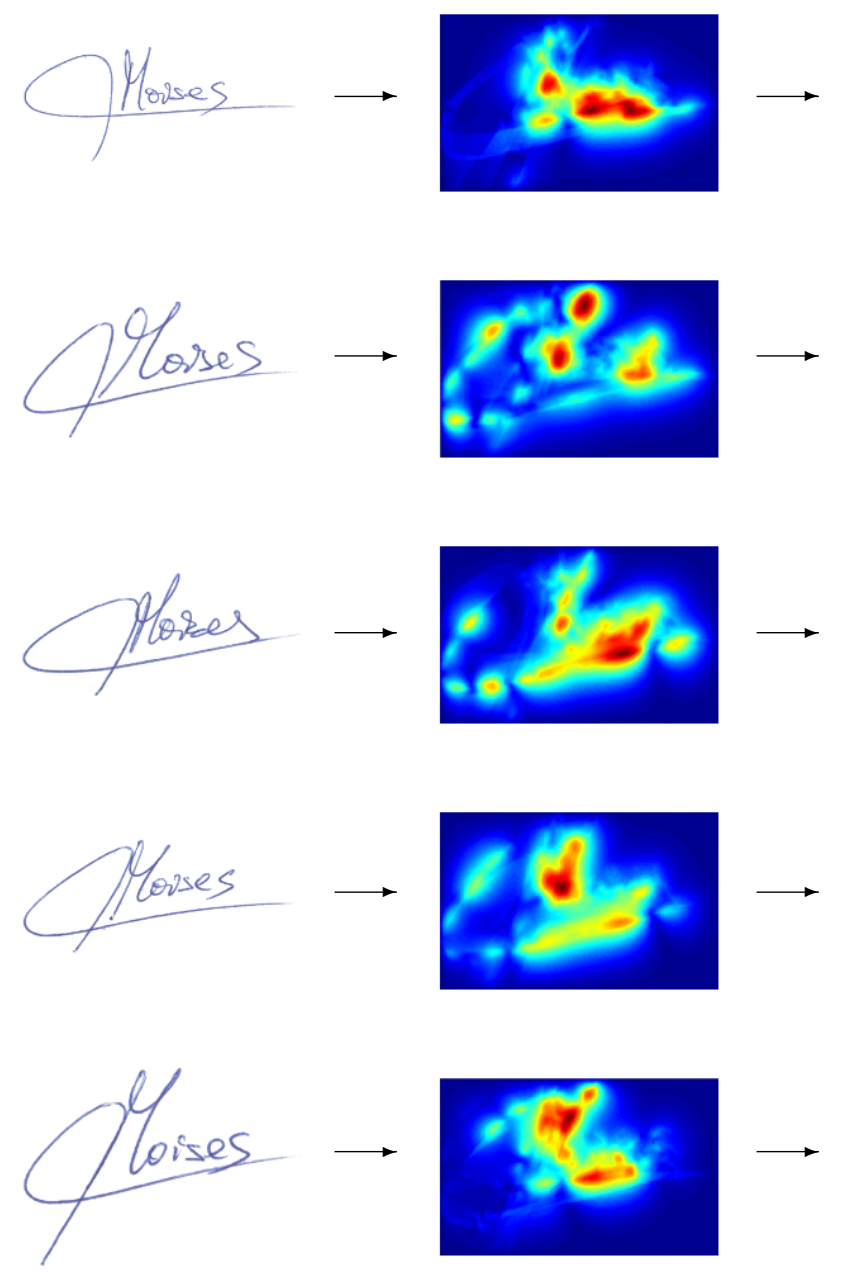

Most Stable areas $S_{i}^{s}$

with $T=10 \%$
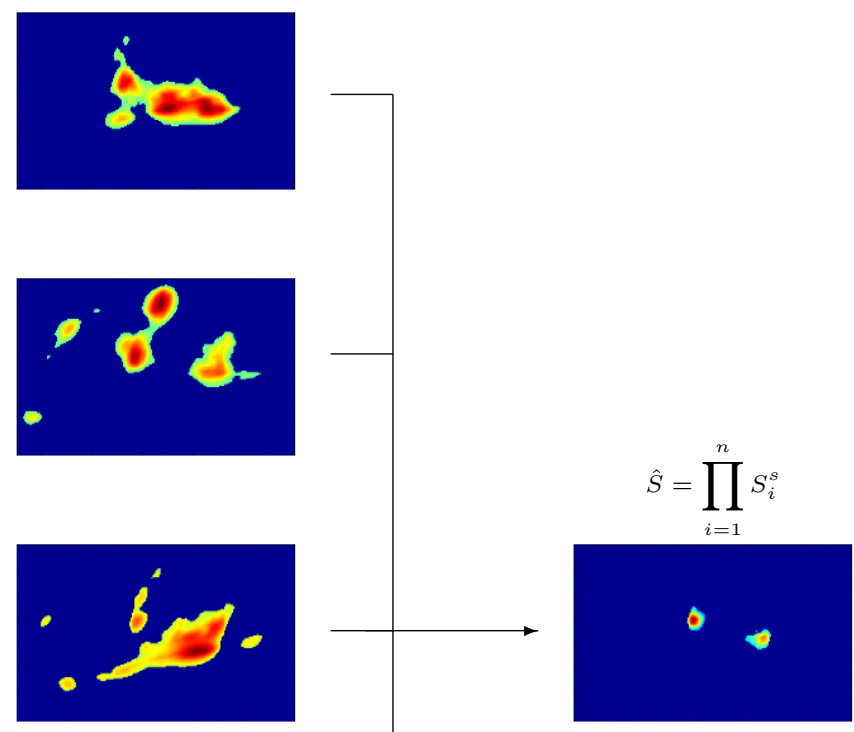

Global Stability scalars: $e_{1}=28.66 \times 10^{-4}$ $e_{2}=0.10$

Fig. 2: Scheme of the process to compute the global stability of a signer.

based, energy-based and phased-based techniques among other are some of the most investigated. In this work the Horn and Shunck proposal [7] is considered. It is based on the minimization of the energy functional in order to obtain an image representing the vector field composed by the displacement vectors calculated between two images. This vector field is so called stability map in this paper.

$$
E=\iint\left[\left(I_{x} u+I_{y} v+I_{t}\right)^{2}+\alpha^{2}\left(\|\nabla u\|^{2}+\|\nabla v\|^{2}\right)\right] d x d y
$$

Assuming that a set of genuine signatures can be considered as the result of different deformation applied to a master signature. This way, the optical flow is worked out to estimate such distortion for each signature. So that, one signature is compared to the rest of the signatures to analyze its particular distortion. In this work this distortion is so called as the local stability of a signature and it is represented as a motion map. In Fig. 2 we could see different local stability maps for each genuine signature of the same signer.
To compute the stability map per signature, firstly we normalized the size of a signature image with a $\alpha$ factor:

$$
\alpha=\sqrt{A /\left(n_{x} \cdot n_{y}\right)}
$$

Where $A$ denotes a fixing area, $n_{x}$ and $n_{y}$ the horizontal and vertical dimensions of the original image. Then, the size of each signature is adapted with $n_{x}^{\prime}=\alpha \cdot n_{x}$ and $n_{y}^{\prime}=\alpha \cdot n_{y}$.

Once the energy functional is calculated through equation (1), the optical flow is deduced in both the horizontal $u$ and vertical components $v$. The last stage computes the local stability map by using the standard deviation from the horizontal and vertical optical flow components with equation (3). Being $\sigma_{u}$ and $\sigma_{v}$ the standard deviation of $u$ and $v$ respectively.

$$
S=\sqrt{\sigma_{u}^{2}+\sigma_{v}^{2}}
$$

We refer the reader to Pirlo et al. 2013 [8] for a detailed explanation of this section. 


\section{Stability ANALYSis For Static Signatures}

This section aims to compute a scalar value to measure the global stability of a signer. As a general formulation, the proposed procedure is based on the following steps: (A) Local stability map; (B) Most stable areas and; (C) Global stability scalars. Fig. 2 illustrates an example of global stability from a set of five genuine signature images.

\section{A. Local stability map}

Formally, Let $\left\{I_{i} \mid i=1, \ldots n\right\}$ be a set of off-line genuine signatures of a signer composed by $n$ specimens in total. Taking one of these signatures as reference, its stability map will be created as follows: After applying the size normalization to the signatures according to (2), the optical flow map is calculated between the reference signature image and one of the rest available ones. This is repeated for the rest of available signatures, obtaining $n-1$ optical flow maps for each comparison. Then, the equation (3) is worked out in order to obtain the local stability maps of the reference signature in each pixel $(\mathrm{x}, \mathrm{y})$. Finally, this procedure is repeated for all remained signatures achieving $n$ local stability maps: $\left\{S_{i} \mid i=1, \ldots n\right\}$

\section{B. Most stables areas}

The most stable areas are extracted from the local stability maps through two segmentation techniques. As such, any pixel from $S_{i}$ that satisfies the condition for the method will be considered part of the most stable area, otherwise will be considered as background. The used method are described as follows:

- Threshold-based: Because it has been used in a large problems of image segmentation during last four decades, the Otsu's Method [9] has been processed. It is based on searching the optimal value to assign a pixel to a class: background or foreground. A threshold is calculated to segment the image in order to minimize the intraclass variance in each class.

- Most illuminated pixels-based: The threshold in this technique is selected according to a $T$ percentage of the most illuminated pixels. In this work, the following values have been studied: $T \in$ $\{5,10,20,30,40,50\} \%$.

In total, seven segmentation methods are analyzed for extracting the most stable areas: one threshold-based and 6 most illuminated pixel-based. At the end of this stage, we will obtain the most stable area maps denoted by $S_{i}^{s}$.

\section{Global Stability scalars}

To measure how stable a signer is, the global stability is calculated through two statistical measures from the global stability map $\hat{S}$.

First of all, the global stability map is computed by the product of all most stable maps of each enrolled signature:

$$
\hat{S}=\prod_{i=1}^{n} S_{i}^{s}
$$

Being $n$ the number of genuine signatures used to compute the stability of a specific user. Next two scalar values are obtained by the entropy and energy of $\hat{S}$.

- Entropy. This statistical method evaluates the global stability map using the information entropy measure designated as:

$$
e_{1}=-\sum_{x y} \hat{S}(x, y) \cdot \log _{2}(\hat{S}(x, y))
$$

This scalar measures statistically the randomness of the stability maps focusing on its texture properties.

- Energy. This statistical evaluates the sum of squared elements in the global stability maps. Its formula is defined by:

$$
e_{2}=\frac{\sum_{x y} \hat{S}^{2}(x, y)}{\left[\sum_{x y} \hat{S}(x, y)\right]^{2}}
$$

The energy $e_{2}$ is normalized in the range $(0,1)$, being $e_{2}=1$ in the case of constant global stability maps.

Finally, different global stability maps along with its entropy and energy are shown at Fig. 3 for different segmentation methods. From Fig. 3-b to 3-g we could observe how the entropy $e_{1}$ decreased when the percentage of illuminated pixels $T$ increases. A direct tendency is observed for the energy, which increases with the parameter $T$. Additionally, it could be also observed that the Otsu's method reports similar values that whose found between $T=20 \%$ and $T=30 \%$.

\section{Automatic OfF-Line Signature Verification}

Two state-of-the-art off-line automatic signature verifiers (ASVs) have been used to evaluate the performance of the system when synthetic samples are introduced. These systems are based on totally different features and classifiers.

- System A: Geometric features + HMM. [10] The signature is parametrized in Cartesian and polar coordinates. Both features are combined at score level. The Cartesian parameters consist of equidistant samples of the height and length of the signature envelope plus the number of times the vertical and horizontal line cut the signature stroke. In polar coordinates the parameters are equidistant samples of the envelope radius plus the stroke area in each sector. A multi observations discrete left to right HMM is chosen to model each signer features. The classification (evaluation), decoding, and training problems are solved with the Forward-Backward algorithm, the Viterbi algorithm, and the Baum-Welch algorithm. The initialization method is the equal-occupancy method.

- System B: Texture features + SVM. [11] This ASV is part of the recent state-of-the-art in signature verification [12]. It is based on texture features such as local binary pattern (LBP) and local derivative pattern (LDP). The signature is transformed into the LBP and LDP images which are divided into 12 sectors. The 


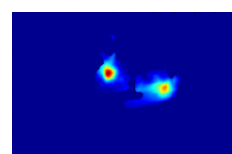

(a) Otsu $e_{1}=7.75 \times 10^{-4}$ $e_{2}=0.38$

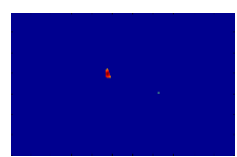

(b) $T=5 \%$

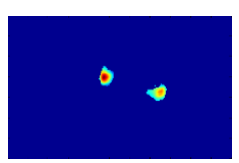

(c) $T=10 \%$

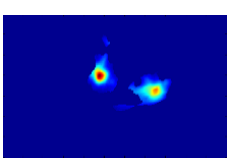

(d) $T=20 \%$

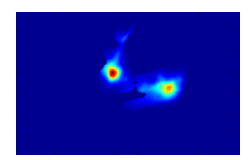

(e) $T=30 \%$ $e_{1}=6.07 \times 10^{-}$ $e_{2}=0.51$

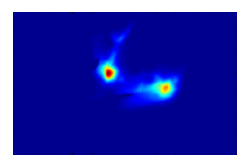

(f) $T=40 \%$ $e_{1}=5.11 \times 10^{-4} \quad e_{1}=4.78 \times 10^{-4}$ $e_{2}=0.71 \quad e_{2}=0.86$

Fig. 3: Global stability maps for different segmentations.

histogram of each sector is worked out, concatenated and its dimension reduced with a Discrete Cosine Transform (DCT) obtaining two separated feature vectors according to LBP and LDP operators. The combination at score level is evaluated through a weighted sum. The classifier is based on a least square support vector machine (LSSVM).

\section{EXPERIMENTS}

\section{A. Database}

In this work the GPDS-100 Offline Signature DB [12] has been used. This dataset contains 23 genuine and 30 deliberated forgeries from first 100 individuals acquired in one site in just one session. Deliberated forgeries mean forged signatures made from certain knowledge of the genuine spatial signature trajectory but it does not necessarily imply acquired skill of a true forger. The repetitions of each genuine signature were collected allowing each donor to use his own pen on sheets of white A4 paper. Each sheet provided two different box sizes for the signature. The sheets were scanned at 600 dpi with 256 grey levels. The database is distributed by the Grupo Procesado Digital de Señales (GPDS) of the ULPGC ${ }^{1}$.

\section{B. Evaluation Methodology}

According to the enrolled signatures, two cases have been studied: the first 5 signatures and the first 8 signatures, following the original order that they were captured. The protocol is conducted as fair as possible and repeatable for others.

On the stability evaluation, we have followed the method explained in Sect. III for both cases of enrolled signatures. In the experiments we have tested all possibilities of segmentation methods as well as the entropy and energy estimators to chose the most representative in each case.

On the performance evaluation, the signatures used as testing set are composed of 15 signatures: from the ninth to the twenty-third. This way, the false rejection rate per signer is calculated using 15 scores. For the random signature test, we have selected the first testing signature of other users, i.e. the genuine signature number nine according to the database nomenclature. We compute the false acceptation rate with 99 scores per user. For the deliberated forgery test, all deliberated available signatures in the databases were used. Therefore, we compute the false acceptation rate per user using 30 scores. The evaluation per signer is computed using the coincident point of the False Acceptation Rate and False Rejection Rate curves. It is so called Equal Error Rate (EER).

\footnotetext{
$1_{\text {www.gpds.ulpgc.es }}$
}

\section{Results}

Once the performance is computed for each user, the users are sorted in ascendant order for each test and each system. In each case, different ascendant curves will be described by the performance. The feasibility of predicting the performance through the stability is observing similar tendencies when the users are sorted by the stability measures. To avoid noisy behavior in the stability curves, they were smoothed with a moving average.

The tested combinations in the global stability representation have been focused on the segmentation methods as well as the scalar values choosing the best combination that defines better the tendency respect to the performance one. The ideal predictor will be achieved whether two curves are overlapped each other.

To clarify the presentation of the curves, the order of the users are shown in ascendant or descendant order regarding to the stability. However, in all cases, the users were sorted in ascendant order according to the performance. It is highlighted with the words "asc." or "des." in Fig. 4 and Fig. 5 accordingly.

On the one hand, the first 5 signatures enrolled in the system lead to observe some relationships according to results in Fig. 4:

- The performance and the stability curves seems to be quite overlapped for System A and System B in the random signature test. In both cases, the statistical value was measured over stability maps with the minimum information considered since the threshold in the segmentation was fixing at $T=5 \%$. It reflects that using only the most important information to build the global stability maps is more convenient to predict the performance tendency in the random signature attack.

- In the case of deliberated signature test a noisy but acceptable relationship is kept between the performance and stability curves in system A. The segmentation method in this case is supposed to use more information from stability maps. While the first and last sorted users seems to be an acceptable relationship, a large offset appears in the rest of the cases, being unclear the findings in the system B.

On the other hand, when the training set is extended to the first 8 signatures, another observations are deduced from Fig. 5:

- Better adjustment is observed in performance and stability in the random test. Again, the best criterion 

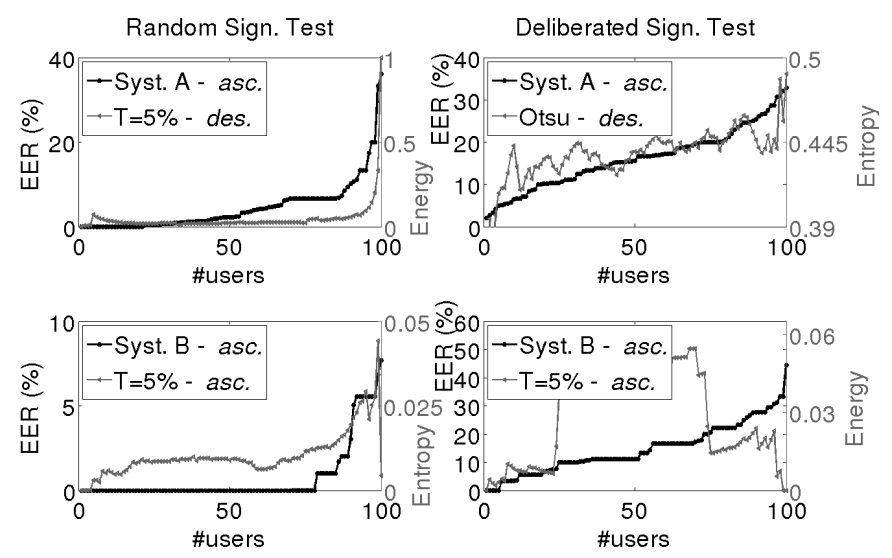

Fig. 4: Relationship between statistical parameters and ERR $(\%)$ per signer when the first 5 signatures are enrolled.

found in the segmentation uses a low information in the global stability maps: $T=10 \%$ and $T=5 \%$ for systems A and B respectively. It could be said that the performance prediction is likely the most accurate in this case.

- Again better results are achieved for the deliberated signature test. Moreover, the adjustment seems to be preferred in the system A instead of system B. It reinforces the behavior studied in this test with the first 5 enrolled signatures. Contrary to the random test, the deliberated test uses a more information in the global stability maps to achieve a more accurate prediction.

\section{CONCLUSION}

In this paper we have studied the stability of the enrolled signature in a system as feasibility measurements to predict the future performance. For its convenience in off-line signatures, optical flow was worked out to represent the stability map for each signature. Different segmentation methods were studied to obtain a global stability map for each user. Then, a statistical global scalar value was analyzed through the entropy and the energy of the global stability map per user. Similarly, the performance of each user was studied through two state-ofthe-art off-line automatic signature verifiers.

The feasibility to predict the performance is assessed by comparison between the performance and the stability of the users. Promising results were found in this preliminary study which suggest that the stability in off-line signatures could predict the future performance of the users using only the $a$ priori information, i.e. without any testing signature. It could be said that the entropy and energy estimators are directly proportional to the performance of the users, which depend on both the tests and the systems. Eventually, the results also show that the more signatures are enrolled, the more refined the prediction will be.

\section{ACKNOWLEDGMENT}

M. D. is supported by a PhD fellowship from the ULPGC. This study was funded by the Spanish governments MCINN TEC2012-38630-C04-02 research project.
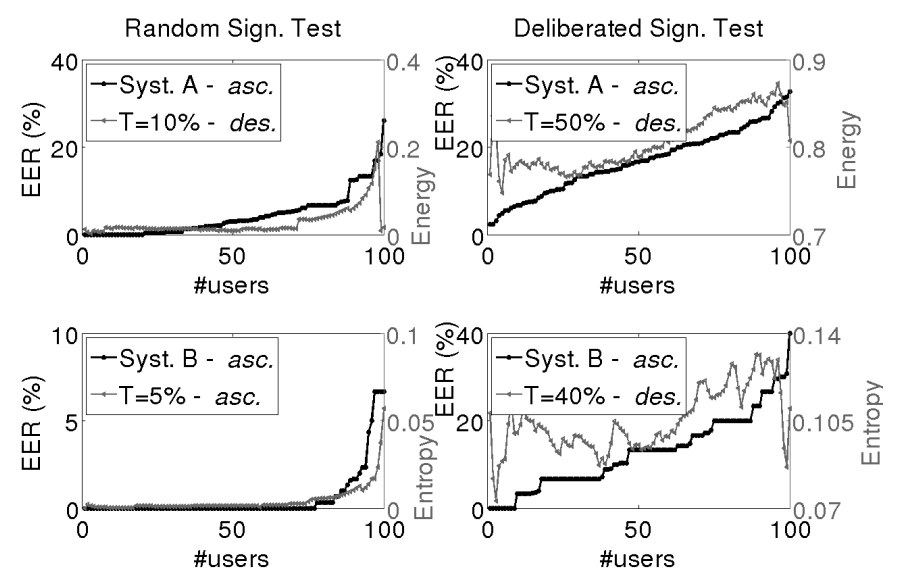

Fig. 5: Relationship between statistical parameters and ERR (\%) per signer when the first 8 signatures are enrolled.

M. D. wishes to thank Prof. Giuseppe Pirlo, professor of Università di Bari, Italy, for hosting him during the development of this research work.

\section{REFERENCES}

[1] M. Diaz-Cabrera, A. Morales, and M. A. Ferrer, Emerging Issues for Static Handwritten Signature Biometric. Word Scientific, 2014, ch. 9, pp. 111-122.

[2] G. Doddington, W. Liggett, A. Martin, M. Przybocki, and D. Reynolds, "Sheep, goats, lambs and wolves a statistical analysis of speaker performance in the NIST 1998 speaker recognition evaluation," in Proc. of International Conference on Spoken Language Processing (ICSLP), 1998, pp. 1351-1354.

[3] M. Diaz-Cabrera, M. A. Ferrer, and A. Morales, "Modeling the lexical morphology of western handwritten signatures," PLOS ONE, vol. 10, no. 4, p. e0123254, 042015.

[4] P. Grother and E. Tabassi, "Performance of biometric quality measures," IEEE Transactions on Pattern Analysis and Machine Intelligence, vol. 29, no. 4, pp. 531-543, 2007.

[5] F. Alonso-Fernandez, M. C. Fairhurst, J. Fierrez, and J. Ortega-Garcia, "Automatic measures for predicting performance in off-line signature," in Intl. Conf. on Image Processing, ICIP, vol. 1, 2007, pp. 369-372.

[6] _-, "Impact of signature legibility and signature type in off-line signature verification," in Biometrics Symposium, BSYM. IEEE, 2007, pp. $1-6$.

[7] B. K. P. Horn and B. G. Schunck, Determining optical flow. MIT Press, A.I. Memo, April 1980, no. 572.

[8] G. Pirlo and D. Impedovo, "Verification of static signatures by optical flow analysis," IEEE Transactions on Human-Machine Systems, vol. 43, no. 5, pp. 499-505, 2013.

[9] N. Otsu, "A threshold selection method from gray-level histograms," IEEE Transactions on Systems, Man and Cybernetics, vol. 9, no. 1, pp. 62-66, Jan 1979.

[10] M. Ferrer, J. Alonso, and C. Travieso, "Offline geometric parameters for automatic signature verification using fixed-point arithmetic," IEEE Transactions on Pattern Analysis and Machine Intelligence, vol. 27, no. 6, pp. 993-997, 2005.

[11] M. Ferrer, J. Vargas, A. Morales, and A. Ordonez, "Robustness of offline signature verification based on gray level features," IEEE Transactions on Information Forensics and Security, vol. 7, no. 3, pp. 966-977, 2012.

[12] M. Blumenstein, M. Ferrer, and J. Vargas, "The 4NSigComp2010 offline signature verification competition: Scenario 2," in Intl. Conf. on Frontiers in Handwriting Recognition, ICFHR, 2010, pp. 721-726. 